\title{
Centromeric Ring and Arrangement of Centromeres in Interphase of Lathyrus sphaericus as Detected by C-banding
}

\author{
U. C. Lavania ${ }^{1}$ and A. K. Sharma \\ Centre of Advanced Studies for Cell and Chromosome Research, \\ University of Calcutta, Calcutta-700019, India
}

Received January 17, 1983

The late prophase orientation of chromosomes is a carryover from late telophase orientation (Carlson 1956). This is apparently maintained by means of the centromeres, which appear to be attached within a limited region of the nucleus throughout telophase, interphase and prophase (DuPraw 1970, Avivi and Feldman 1980, Lavania and Sharma 1981). Very little is known about the arrangement of centromeres in an interphase nucleus, owing to the inherent technical difficulty to recognize the centromeres at interphase under light or electron microscope.

In Lathyrus sphaericus, C-bands as detected by Giemsa banding have been found to be present mainly in centromeric region as thick blocks of C-heterochromation. An additional very thin band is also observed in satellited regions of the nucleolar pair (Lavania and Sharma 1980a). Thus Lathyrus sphaericus provides a suitable material for the study of arrangement, orientation and behaviour of centromeres during cell division cycle.

\section{Material and methods}

Seeds of Lathyrus sphaericus Retz. were sown at $20^{\circ} \mathrm{C}$. Fast growing root tips were directly fixed in Carnoy's fixative so as to arrest the cells at various stages of the cell cycle for the study of C-band distribution and behaviour of centromeres. For C-banding, the procedure of Vosa and Marchi (1972) for plants was followed.

\section{Observations}

\section{C-band distribution at interphase}

Observations were recorded on a monolayer group of interphase cells of the root tip squashes, rather than on too much scattered and isolated cells, to minimise the effect of pressure applied during squashing on the possible displacement of centromeres. The data were analysed from 5 slides (one root tip made into 5 pieces per slide) at five different locations in each case. Giemsa positive dark stained dots obtained after the C-banding technique were observed to be arranged in the form of a ring in interphase nucleus. Nearly, all such cells, revealed ring-like arrange-

1 Present address: Central Institute of Medicinal and Aromatic Plants, Post Bag No. 1, Post- Ram Sagar Misra Nagar, Lucknow-226016, India. 
ment of centromeres. Out of a total of 200 interphase cells showing a distinct ringlike arrangement of C-band regions examined, $16.5 \%$ showed a few C-band dots in the central gap of the ring (Fig. 1). This ring of heterochromatic regions was formed in one of the polar region of the nucleus. With the advance of interphase (as revealed from the nuclear size and heterochromation amount; 2C-to-4C transition), the $\mathrm{C}$-band regions of the ring fuse in a group of 2-3 bands (as apparent from the size) to form 5-7 thick blocks of heterochromation at the centromere region (Fig. 2). The number and size of these centromeric heterochromtaic groups at interphase vary between nuclei.

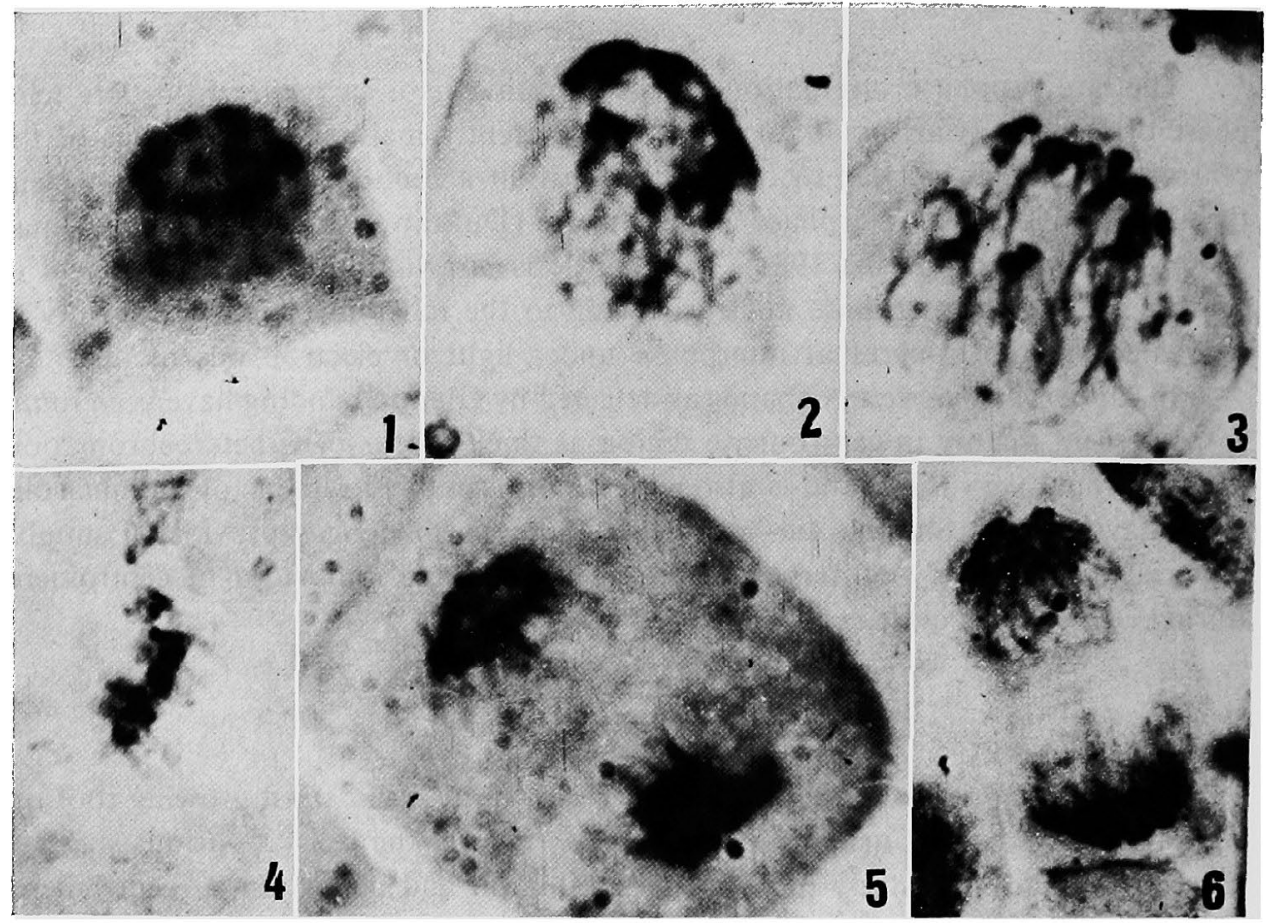

Figs. 1-6. 1, centromeric ring at interphase. Note, few Giemsa positive dots in the central gap of the ring. 2, centromeric groupings at interphase. 3, late prophase nucleus. Note, fusion of centromeres in some cases. 4, C-banded metaphase at equitorial plate. 5, anaphase. Note, ringlike arrangement of centromeres. 6, two sister cells at early prophase. Note, polarizaed nucleus and ring-like arrangement of Giemsa stained regions on abaxial poles.

\section{Orientation of $\mathrm{C}$-band regions during mitotic movements}

With the transition from interphase to prophase and the reorganization of the chromosomes, the separate C-band regions are also resolved. However, the Cband regions still maintain the ring-like arrangement and chromosomes appear to remain polarized until late prophase (Fig. 3). At metaphase, the C-banded segments are arranged at the equatorial plate (Fig. 4). However, in some plates, where the chromosomes show radial metaphase like arrangement, the $\mathrm{C}$-band regions form ring-like configuration. When the sister chromatids separate and move towards 
opposite poles at anaphase, centromeric regions form a ring-like arrangement as evidenced by dark stained C-band regions (Fig. 5). A similar arrangement is retained at telophase and subsequently at interphase (Fig. 6). All these observations clearly show that the arrangement of chromosomes is non-random throughout mitosis and the centromeric regions maintain their ring-like arrangement all over the division cycle as exhibited by arrangement and orientation of $\mathrm{C}$-banded centromeric regions.

\section{Discussion}

\section{Arrangement of chromosomes in interphase and C-band distribution during mitotic movements}

Constitutive heterochromation as detected by Giemsa C-banding technique is mainly confined to centromeric regions of chromosomes in L. sphaericus. At various stages of mitotic cell cycle in this species, the $\mathrm{C}$-band regions form a ring-like configuration showing thereby that anaphase-telophase orientation of chromosomes is maintained at interphase and even till late prophase. Previous data on cytology (Avivi and Feldman 1980), autoradiography (Fussell 1975), electron microscopy (Church and Moens 1976) and banding (Stack and Clark 1974, Fussell 1977, Ghosh and Roy 1977, Tanaka and Tanaka 1977, Lavania and Sharma 1980b) studies on a variety of plants show that interphase chromosomes retain their telophase arrangement. However, Hsu et al. (1971) found that position of centromeres in the interphase nucleus of Mus musculus varied from tissue to tissue. Fussell (1977) suggested that telophase orientation of interphase chromosomes is typical of cells with high mitotic rates. This non-random telophase arrangement of interphase chromosomes possibly determines the plane of division.

The centromeric regions of chromosomes as revealed by C-bands remain polarized in the abaxial hemisphere of the nucleus and possibly the telomeric ends in other hemisphere. This polarization is a relic of anaphase-telophase chromosome orientation from the preceding division.

\section{Centromeric ring}

As evident from the observations, the ring-like configuration of centromeric heterochromatic regions is maintained throughout the division cycle. Even in metaphase plates where the chromosomes are slightly separated from each other and some radial metaphase like arrangement becomes apparent, the $\mathrm{C}$-band regions can be seen arranged in a ring.

Mosolov (1974) and Mosolov and Bondareva (1976) in their models have suggested that in the interphase nucleus chromosomes are meridionially arranged. The centromeres are concentrated on one of the polar circles forming a peculiar polar ring (centromeric ring). In view of this fact it appears that centromeric pole is most clearly expressed on the interphase nucleus and the telomeres are oriented towards the opposite pole. The centromeric ring unites the chromosomes in the region of centromere and is responsible for orienting them at all stages of the cell cycle. The so-called centromeric ring is a mechanism maintaining polar orientation 
of chromosomes. Mosolov (1974) suggested that the chromosomes always maintain a strictly non-random arrangement, independent of the cell cycle. Only by preserving this constancy, the regular spatial relationship of the genetic apparatus in the dividing cells is possible.

Yunis and Yasmineh (1971) suggested that the grouping of centromeres in a cell is a function of centric heterochromation which has an aggregation property. As the interphase cell advances, the heterochromatic regions of the centromeric ring aggregate to form 5-7 centromeric structures. Similar aggregation of centromeric regions in mitotic and meiotic interphase nuclei has been reported in Allium fistulosum (Church and Moens 1976, Moens and Church 1977). In some of the interphase cells a few C-bands could be seen in the central gap of the centromeric ring. These may be ascribed to the $\mathrm{C}$-bands of nucleolar segments. That the interstitial C-bands of nucleolar chromosomes remain in the nucleoplasm has been reported in Allium cepa and Trigonella foenum-graecum from the observations of Cbands and electron micrographs of interphase nucleus (Ghosh and Roy 1977, Lavania and Sharma 1980b). This ring-like arrangement of centromeres in interphase nucleus has also been noted by the present authors in Lathyrus odoratus, as having almost a similar type of C-banding pattern. Avivi and Feldman (1980) reviewed the arrangement of chromosomes in plants and inferred a circular arrangement of centromeres in interphase nucleus.

The mechanism of maintaining this chromosome orientation is not fully known. One possibility is that heterochromatic regions rich in repetitive DNA and specific non-histones may serve as points of attachment to nuclear membrance (Sharma 1978, Lavania and Sharma 1981, 1982) which may well provide a mechanism for holding chromosomes in place within the nucleus (Fussell 1975, 1977, Avivi and Feldman 1980, Comings 1980). The existence of chromosomal inter-connection by thin filaments of chromatin running from one chromosome to the next during mitotic and meiotic metaphases has been clearly demonstrated in various cell types through different approaches (Hoskins 1968, Klasterska et al. 1977, Lavania and Sharma 1984). These interchromosomal connections may be involved in maintaining a spatial relation among chromosomes and in governing the exact distribution of the chromosomes at all stages of cell division.

\section{Summary}

The C-bands of Lathyrus sphaericus are present in the centromeric region of all the 14 chromosomes and the satellited region of the nucleolar pair. Orientation of interphase chromosomes is non-random and late anaphase arrangement of chromosomes is maintained up to next late prophase through interphase. At interphase, centromeric regions form a ring-like arrangement towards one pole and chromosomes remain polarised. The chromosomes may be present singly or 2-3 centromeres may associate to form 5-7 centromeric masses. Regularity of spatial relationshipof chromosomes throughout the division cycle is indicated. Non-random arrangement of interphase chromosomes and organization of a structure like centromeric ring for maintaining the spatial relationship have been demonstrated. 


\section{Acknowledgements}

This work is financed by a teacher fellowship grant awarded to one of us (UCL) by the University Grants Commission.

\section{References}

Avivi, L. and Feldman, M. 1980. Arrangement of chromosomes in the interphase nucleus of plants. Hum. Genet. 55: 281-295.

Carlson, J. G. 1956. On the mitotic movements of chromosomes. Science 124: 203-206.

Church, K. and Moens, P. B. 1976. Centromere behaviour during interphase and meiotic prophase in Allium fistulosum from 3-D, E.M. resconstruction. Chromosoma 56: 249-263.

Comings, D. E. 1980. Arrangement of chromatin in the nucleus. Hum. Genet. 53: 131-143.

DuPraw, E. J. 1970. DNA and Chromosomes. Holt, Rinehart \& Winston Inc.

Fussell, C. P. 1975. The position of interphase chromosomes and late replicating DNA in centromeric and telomeric regions of Allium cepa (L.). Chromosoma 50: 201-210.

- 1977. Telomere association in interphase nuclei of Allium cepa demonstrated by C-banding. Exp. Cell Res. 110: 111-117.

Ghosh, S. and Roy, S. C. 1977. Orientation of interphase chromosomes as detected by Giemsa C-bands. Chromosoma 61 : 49-55.

Hoskins, G. C. 1968. Sensitivity of micro-surgically removed chromosomal spindle fibres to enzyme disruption. Nature 217: 748-750.

Hsu, T. C., Cooper, J. E. K., Mace, M. L. and Brinkley, B. R. 1971. Arrangement of centromeres in mouse cells. Chromosoma 34: 73-87.

Klasterska, I., Natarajan, A. T. and Ramel, C. 1977. Interchromosomal connections in mouse spermatocyte meiosis. Hereditas 86: 273-276.

Lavania, U. C. and Sharma, A. K. 1980a. Giemsa C-banding in Lathyrus L. Bot. Gaz. 141: 199-203.

- and - 1980b. Giemsa C-banding, somatic association and orientation of interphase chromosomes in Trigonella foenum-graecum (L.). Caryologia 33: 17-23.

- and - 1981. An interphase model for mitotic chromosome organization in Eukaryota. Biosystems 14: 171-178.

- and - 1982. Heterochromatin in perspective. Curr. Sci. 51: 175-180.

- and - 1984. On the interchromosomal connections in plants. Experientia 40: 94-95.

Moens, P. B. and Church, K. 1977. Centromere sizes, positions and movements in the interphase nucleus. Chromosoma 61 : 41-48.

Mosolov, A. N. 1974. Evolutional conception of nucleus and chromosome structure. Nucleus 17: $51-64$.

- and Bondareva, A. A. 1976. Achromatic pole, centromeric ring and nucleolus-Spatial interrelation in mitotic and interphase nucleus. Ibid. 19: 115-123.

Sharma, A. K. 1978. Additional genetic elements in chromosomes. Ibid. 21: 113-116.

Stack, S. M. and Clark, C. R. 1974. Chromosome polarization and nuclear rotaion in Allium cepa roots. Cytologia 39: 553-560.

Tanaka, N. and Tanaka, N. 1977. Behaviour of differentially stained kinetochores during the mitotic cell cycle in some Polygonatum species (Liliaceae). Ibid. 42: 765-775.

Vosa, C. G. and Marchi, P. 1972. Quinacrine fluorescence and Giemsa staining in plants. Nature (New Biol.) 237: 191-192.

Yunis, J. J. and Yasmineh, W. G. 1971. Heterochromation, satellite DNA and cell function. Science 174: 1200-1209. 